UDC 539

\title{
Probe diagnostics of plasma generated by a volume source of fission fragments in the active zone of stationary nuclear reactor WWR-K
}

\author{
Kunakov S. ${ }^{1}$, Son E. ${ }^{2}$, Shapiyeva A. ${ }^{1}$ \\ ${ }^{1}$ International University of Information Technologies, \\ 34 "A"/8 "A" Manasa/Zhandosov str., 050040 Almaty, Kazakhstan \\ ${ }^{2}$ Joint Institute of High Temperature AS, Russian Federation \\ e-mail: sandybeck.kunakov@gmail.com
}

\begin{abstract}
This paper presents the theory and the experimental results of probe diagnostics for high-pressure nuclear induced plasma in the presence of negative ions and mathematical description based on the hydrodynamic approximation and methodological recommendations are given to define that concentrations of charged particles in the plasma on the basis of numerical calculations. ${ }^{3} \mathrm{He}+U F_{6}$ plasma is generated by the nuclear reaction products ${ }^{3} \mathrm{He}+n+p \rightarrow p+T+0.76 \mathrm{MeV}$.
\end{abstract}

Key words: probe diagnostics, helium plasma, fission fragments, nuclear reactor.

PACS number(s): 28.41.-i

\section{Introduction}

The probe method is easy to implement and commonly used as an effective local experimental tool among plasma diagnostics methods. Nevertheless, the correct theoretical description of the electric currents formation in the region disturbed by electric probe is very complicated. As it's well known, the current-voltage characteristics (CVC) are used to define the concentration of charged particles and electrons temperature. The degree of ionization in nuclear induced plasma is supposed to be small $N_{e} \leq N_{0}$, where $N_{0}$ - the concentration of neutral atoms) and the frequency of electron collisions with electrons and ions in plasma is assumed to be negligible small compared with the electrons collisions with neutrals. The electron free flight length as well as the ions length is assumed to be much more less then the charged particles characteristic length. These physical conditions imply that the plasma is collisional and might be described in the hydrodynamical approximation. The major part of the experimental results implemented in nuclear test-reactor WWR-K is presented in paper [1].

The theory of electrostatic probes in the not-self-maintained plasma for the probes of different geometry and for the weakly ionized plasma of high-pressure flow were developed by Ulyanov [2], [3]. In this paper the disturbed zone was subdivided into the two zones: the region of charged layer, the region of diffusion layer.
In the paper [4] the asymptotic theory of spherical electrostatic probe is presented for the case of chemically active, weakly ionized high-pressure plasma in the presence of small concentrations of negative ions. It is also assumed that Debye radius is small compared with the probe size, and electron energetic length of relaxation is much more smaller then the length of microscopic scale, and function of electrons energy distribution is defined by local magnitudes of electron density and temperature.

2 The theory of electrostatic probe in nuclear induced plasma with negative ions

\subsection{Basic equations}

We take the case of stationary plasma and the probe potential is different from the plasma potential. We shall take the distance from the probe till any point of disturbed by the probe region as a basic coordinate. The non dimensionless basic equations describing the formation of electron and ion currents might be presented in the following way:

$$
\begin{gathered}
\omega^{+} \frac{d}{d \xi}\left(-\frac{d n^{+}}{d \xi}+\gamma n^{+} \frac{d \Psi}{d \xi}\right)=1-a n^{e} n^{+}-b n^{-} n^{+} \\
\omega^{-} \frac{d}{d \xi}\left(-\frac{d n^{-}}{d \xi}+\gamma n^{-} \frac{d \Psi}{d \xi}\right)=c n^{e}-b n^{-} n^{+}
\end{gathered}
$$




$$
\begin{gathered}
\omega^{e} \frac{d}{d \xi}\left(-\frac{d n^{e}}{d \xi}+n^{e} \frac{d \Psi}{d \xi}\right)=1-c n^{e}-a n^{e} n^{+} \\
\varepsilon \frac{d^{2} \Psi}{d \xi^{2}}=n^{e}-n^{-}+n^{+}
\end{gathered}
$$

where $\xi=\frac{r}{r_{p}}, \quad \omega^{+}=\frac{t_{i o n}}{t_{D^{+}}}, \quad \omega^{-}=\frac{t_{i o n}}{t_{D^{-}}}, \quad \omega^{e}=\frac{t_{i o n}}{t_{D^{e}}}$, $t_{\text {ion }}=\frac{n_{\infty}}{S}, \quad t_{D_{+}}=\frac{r_{p}^{2}}{D_{+}}, \quad t_{D_{e}}=\frac{r_{p}^{2}}{D_{e}}, \quad t_{D_{-}}=\frac{r_{p}^{2}}{D_{-}}$, $\varepsilon=\left(\frac{r_{d}}{r_{p}}\right)^{2}=\frac{K T_{e}}{4 \pi e^{2} n_{\infty}^{+} r_{p}^{2}}, \quad \Psi=\frac{\varphi}{\frac{k T_{e}}{e}}, \quad a=\frac{\alpha_{e}\left(n_{\infty}^{+}\right)^{2}}{S}$, $b=\frac{\alpha_{i}\left(n_{\infty}^{+}\right)^{2}}{S}, \quad c=\frac{\beta n_{\infty}^{+}}{S}, \quad \gamma=\frac{T_{e}}{T}, \quad \delta=\frac{n_{\infty}^{e}}{n_{\infty}^{+}}$, $S=\frac{n_{\mathrm{He}_{3}} * \Phi * \sigma_{f} * E_{0}}{\Omega}$,

$n^{+}, n^{-}, n^{e}$ - concentration of electrons, positive and negative ions, $D^{+}, D^{-}, D^{e}, b^{+}, b^{-}, b^{e}$ - coefficients of diffusion and mobility of electrons, negative and positive ions, $\beta$ - affinity coefficient [4] defining the formation of negative ions, $r_{p}$ - probe radius, $S-$ ionization rate, $\Phi$ - neutron flux density, $\sigma_{f}-$ fission cross section of the helium-3, $E_{0}$ - the kinetic energy of the fission fragments, $\Omega$ - the energy value of the formation of an electron an ion pair, $n_{\mathrm{He}_{3}}-$ the helium-3 concentration.

Boundary conditions on the probe and on the limits of disturbed region are defined as follows:

$$
\begin{gathered}
n^{+}(1)=n^{-}(1)=n^{e}(1)=0, \Psi\left(r_{p}\right)=\Psi_{p}, \\
n^{+}\left(\xi_{1}\right)=1, n^{-}\left(\xi_{1}\right)=1-\delta, n^{e}\left(\xi_{1}\right)=\delta, \Psi\left(\xi_{1}\right)=0,
\end{gathered}
$$

where $\xi_{1}$ - the length of disturbed region.

\subsection{Charged layer}

Following the results of work [2], we divide the disturbed region into two layers: the charged layer $1 \leq \xi \leq \xi_{0}$ and the layer of ambipolar diffusion $\xi_{0} \leq \xi \leq \xi_{1}$.

In the charged layer the equations which form the probe currents of positive and negative particles presented as follows:

$$
\omega^{+} \frac{d}{d \xi}\left(\gamma n^{+} \frac{d \Psi}{d \xi}\right)=1
$$

$$
\begin{gathered}
\omega^{-} \frac{d}{d \xi}\left(\gamma n^{-} \frac{d \Psi}{d \xi}\right)=0, \\
\omega^{e} \frac{d}{d \xi}\left(n^{e} \frac{d \Psi}{d \xi}\right)=1, \\
\varepsilon \frac{d^{2} \Psi}{d \xi^{2}}=n^{e}-n^{-}+n^{+} .
\end{gathered}
$$

From equations (7-10) the lengths of charged layer for the plane, cylindrical and spherical probes will be presented like these:

for plane probe:

$$
r_{0}=r_{p}\left[1+\kappa_{+}^{-\frac{1}{2}} r_{p}^{-2}\left(\phi_{p}-\phi_{0}\right)\right]^{\frac{1}{2}},
$$

for cylindrical probe:

$$
r_{0}=r_{p}\left[1+\sqrt{2} \kappa_{+}^{-\frac{1}{2}} r_{p}^{-2}\left(\phi_{p}-\phi_{0}\right)\right]^{\frac{1}{2}},
$$

for spherical probe:

$$
r_{0}=r_{p}\left[1+\sqrt{3} \kappa_{+}^{-\frac{1}{2}} r_{p}^{-2}\left(\phi_{p}-\phi_{0}\right)\right]^{\frac{1}{2}},
$$

where $\kappa_{+}=\left(\frac{4 \pi e S}{b_{+}}\right)^{\frac{1}{2}}$.

For the negative probe potential the electric currents on the plane, cylindrical and spherical probes are presented as follows:

for plane probe:

$$
I_{p}=e S A r_{0},
$$

for cylindrical probe:

$$
I_{p}=e S \frac{\phi_{p}-\phi_{0}}{\sqrt{\kappa_{+}}} L,
$$

for spherical probe:

$$
I_{p}=\frac{4}{3} \pi e S r_{p}^{3}\left[1+\sqrt{\frac{3}{\kappa_{+}}} \frac{\phi_{p}-\phi_{0}}{r_{p}^{2}}\right]^{\frac{3}{2}},
$$

where A - plane probe area, L - length of cylindrical probe, and the lengths of charged layer for different geometry might be presented as follows:

If the full voltage drop is in the charged layer then following to [3] we have the simple way of charged particle concentration definition. This method is based on the use of linear part of electronic branch of CVC near the zero. Parameter $\delta$ varies from $0 \leq \delta \leq 1$. This an equality describes limited cases when plasma consists of only negative and positive ions or electrons and positive ions. 
In the case when voltage drop is taken place in the charged layer the concentration of positive ions and parameter $\delta$ is defined from ion and electron branches of $\mathrm{CVC}$ by the following expressions (spherical probe) [5]:

$$
\begin{gathered}
n_{\infty}^{+}=\frac{J_{n p}^{+}\left(1+\frac{3}{4} \frac{I_{n p}}{\pi e S r_{p}^{3}}\right)^{\frac{1}{3}}-1}{\varphi_{n p} r_{p}\left(1+\frac{3}{4} \frac{I_{n p}}{\pi e S r_{p}^{3}}\right)^{\frac{2}{3}}}, \\
\delta=\frac{J_{n p}^{-}-4 \pi e r_{p} b^{-} n_{\infty}^{+} \varphi_{p p}\left(1+\frac{J_{n p}^{-}}{4 \pi e S r_{p}^{3}}\right)^{\frac{1}{3}}}{4 \pi e r_{p}\left(b^{e}-b^{-}\right) \varphi_{p p}\left(1+\frac{J_{n p}^{-}}{4 \pi e S r_{p}^{3}}\right)^{\frac{1}{3}}}
\end{gathered}
$$

where $J_{n p}^{+}, \varphi_{n p}$ - probe current and negative probe potential, $J_{n p}, \varphi_{n p}$ - probe current and positive probe potential.

\subsection{Ambipolar diffusion layer}

Basic equations in diffusion layer:

$$
\begin{gathered}
\omega_{a}^{+} \frac{d}{d \xi}\left(-\frac{d n^{+}}{d \xi}\right)=1-a n^{e} n^{+}-b n^{-} n^{+} \\
\omega_{a}^{-} \frac{d}{d \xi}\left(-\frac{d n^{-}}{d \xi}\right)=c n^{e}-b n^{-} n^{+} \\
\omega_{a}^{e} \frac{d}{d \xi}\left(-\frac{d n^{e}}{d \xi}\right)=1-c n^{e}-a n^{e} n^{+} \\
\varepsilon \frac{d^{2} \Psi}{d \xi^{2}}=n^{e}-n^{-}+n^{+}
\end{gathered}
$$

where $\omega_{a}^{+}=\frac{t_{i o n}}{t_{D_{a}^{+}}}, \omega_{a}^{-}=\frac{t_{i o n}}{t_{D_{a}^{-}}}, \omega_{a}^{e}=\frac{t_{i o n}}{t_{D_{a}^{e}}}$.
Everywhere faraway from the probe the following relations are valid:

$$
\begin{gathered}
J^{+}-J^{-}-J^{e}=0, \\
J_{p}=e J^{+}+e Z J^{-}+e J^{e}, \\
\frac{d \varphi}{d x}=-\left(\frac{D^{+}-(1-\delta) D^{-}-\delta D^{e}}{b^{+}+(1-\delta) b^{-}+\delta b^{e}}\right) \frac{d \ln \left(n^{+}\right)}{d x}, \\
J^{+}=-D_{a}^{+} \frac{d n^{+}}{d x} \\
J^{-}=-D_{a}^{-} \frac{d n^{-}}{d x}
\end{gathered}
$$

$$
J^{e}=-D_{a}^{e} \frac{d n^{e}}{d x}
$$

where $D_{a}^{+}, D_{a}^{-}, D_{a}^{e}-$ ambipolar diffusion coefficients:

$$
\begin{gathered}
D_{a}^{+}=\frac{\delta\left(D^{+} b^{e}+D^{e} b^{+}\right)+(1-\delta)\left(D^{+} b^{-}+D^{-} b^{+}\right)}{b^{+}+(1-\delta) b^{-}+\delta b^{e}}, \\
D_{a}^{-}=\frac{\left(D^{+} b^{-}+D^{-} b^{+}\right)+\delta\left(D^{+} b^{e}+D^{e} b^{-}\right)}{b^{+}+(1-\delta) b^{-}+\delta b^{e}} \\
D_{a}^{+}=\frac{\left(D^{+} b^{e}+D^{e} b^{+}\right)}{b^{+}+(1-\delta) b^{-}+\delta b^{e}} .
\end{gathered}
$$

In diffusion layer for negative probe potentials electric currents for plane, cylindrical and spherical geometry are as follows:

for plane probe:

$$
I_{p}=e\left(1+\frac{b_{+}}{b_{+}+(1-\delta) b_{-}+\delta b_{e}}\right) D_{a}^{+} n_{\infty}^{+} r_{p}^{-1}\left[\frac{2}{\omega_{+}}\left(1-\frac{\rho}{3}\right)\right]^{\frac{1}{2}} A
$$

for cylindrical probe:

$$
I_{p}=2 \pi e\left(1+\frac{b_{+}}{b_{+}+(1-\delta) b_{-}+\delta b_{e}}\right) D_{a}^{+} n_{\infty}^{+} L\left[\frac{2}{\omega_{+}}\left(1-\frac{\rho}{3}\right)\right]^{\frac{1}{2}}\left(1+\frac{\phi_{p}-\phi_{0}}{\sqrt{\kappa} r_{p}^{2}}\right)^{\frac{1}{2}}
$$

for spherical probe:

$$
I_{p}=\pi e\left(1+\frac{b_{+}}{b_{+}+(1-\delta) b_{-}+\delta b_{e}}\right) D_{a}^{+} n_{\infty}^{+} r_{p}\left[\frac{2}{\omega_{+}}\left(1-\frac{\rho}{3}\right)\right]^{\frac{1}{2}}\left(1+\frac{\phi_{p}-\phi_{0}}{\sqrt{\kappa} r_{p}^{2}}\right)^{\frac{1}{2}} .
$$


The value $n_{0}$ of boundary concentration for the negative and positive probe is defined by the following:

$$
\begin{gathered}
\left(\frac{r_{p}}{r_{d}}\right)^{2} n_{0}^{+}=\vartheta\left[\frac { 2 } { \omega _ { + } } \frac { 1 } { ( n _ { 0 } ^ { + } ) ^ { 2 } } \left(1-\frac{\rho}{3}-n_{0}^{+}+\right.\right. \\
\left.\left.+\frac{\rho\left(n_{0}^{+}\right)^{3}}{3}\right)-\frac{1}{n_{0}^{+}}\left(1-\rho\left(n_{0}^{+}\right)^{2}\right)\right], \\
\left(\frac{r_{p}}{r_{d}}\right)^{2} n_{0}^{e}=\vartheta\left[\frac { 2 } { \omega _ { e } } \frac { 1 } { ( n _ { 0 } ^ { e } ) ^ { 2 } } \left(\delta-\frac{\delta \rho}{3}-n_{0}^{e}+\right.\right. \\
\left.\left.+\frac{\rho\left(n_{0}^{e}\right)^{3}}{3}\right)-\frac{1}{n_{0}^{e}}\left(1-\rho\left(n_{0}^{e}\right)^{2}\right)\right] .
\end{gathered},
$$

If the main potential drop is taken place in diffusion zone then we have the following:

$$
\begin{aligned}
& n_{0}^{+}=\left(\frac{\vartheta \varepsilon}{\omega_{+}}\right)^{\frac{1}{3}}\left[2\left(1-\frac{1}{3} \rho\right)\right]^{\frac{1}{3}}, \\
& n_{0}^{e}=\left(\frac{\vartheta \varepsilon}{\omega_{+}}\right)^{\frac{1}{3}}\left[2 \delta\left(1-\frac{1}{3} \rho\right)\right]^{\frac{1}{3}},
\end{aligned}
$$

where $\vartheta=\frac{D_{+}-(1-\delta) D_{-}-\delta D_{e}}{b_{+}+(1-\delta) b_{-}+\delta b_{e}}\left(\frac{k T_{e}}{e}\right)^{-1}$, $\omega_{+}=\frac{D_{a+} n_{+\infty}^{2}}{S r_{p}^{2}}, \omega_{e}=\frac{D_{a e} n_{+\infty}^{2}}{S r_{p}^{2}}, \varepsilon=\left(\frac{r_{d}}{r_{p}}\right)^{2}$, $\rho=\frac{\alpha_{e} n_{+\infty}^{2}}{S} \delta+\frac{\alpha_{i} n_{+\infty}^{2}}{S}(1-\delta), \quad \delta=\frac{n_{\infty}^{e}}{n_{\infty}^{+}}$.
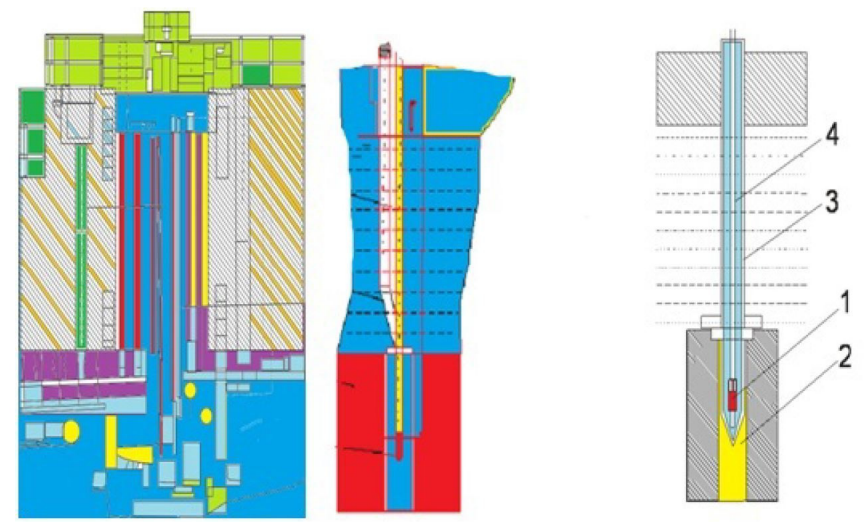

Figure 1 - WWR-K reactor scheme, diagnostic channel and diagnostic cell ( 1 - testing ampule, 2 - active zone, 3 - diagnostic channel, 4 - signal wires))
3 The electrostatic probe experimental data obtained in plasma ${ }^{3} \mathrm{He}+U \mathrm{~F}_{6}$ diagnostics in the active zone of nuclear reactor WWR-K

WWR-K reactor is research test-reactor working on thermal neutrons. Moderator as well as reflector of neutron flux as well as the cooler is artesian water desalinated by ion-exchange absorber AB-17, KU-2. Active zone consists from assembly of uranium fuel elements which are placed in hexagonal space symmetry. The high of the test-reactor is about $60 \mathrm{~cm}$. The cooling system of the reactor is made by two independent cooling cycles. The cooler of the first cycle is transferring the heat of fuel elements with the speed $5 \mathrm{~m} / \mathrm{s}^{-1}$, and the volume rate 1600 $\mathrm{m}^{3} / \mathrm{h}$. The cooling temperature is maintained on the constant level and does not exceed $400{ }^{\circ} \mathrm{C}$.

The vertical projection of the active zone is presented on the figure 1a. The active zone is set in the vessel with artesian water and surrounded by biological protection shield made from the cast iron and heavy concrete. The water layer over the active zone is about 4 meters. The top of the apparatus is closed by rotating iron cover with a bung, through which the core services, loading and unloading through the vertical experimental channel samples and testing products are carried out.

The central testing channel of active zone is located in the beryllium moderator which is set in the centre of active zone replacing four or seven fuel elements (which corresponds to the diameter testing channels $96 \mathrm{~mm}$ and $140 \mathrm{~mm}$ ). The reactor experiments are specific and the operation with experimental sets are connected with the ionising radiation. 
The main specific feature of reactor experiments is that the experimental measurements control might be done only remotely and the experimental set might be used only single time. Due to the high induced radiation the experimental construction elements are not available. The construction elements as well as the optical parts should be highly resistant to the radiation flux. As a source of radiation the ${ }^{3} \mathrm{He}$ isotope is used which has large cross-section value with thermal neutrons $5.3 \cdot 10-21 \mathrm{~cm}^{2}(5.3 *$ $10-21 \mathrm{~cm} 2)$ and the undergoing products of this reaction $\operatorname{He}(n, p) T$ will cause the ionization of the testing gas mixture:

$$
{ }^{3} \mathrm{He}+n \rightarrow p+T+(0.76 \mathrm{MeV}),
$$

The input energy per unit of volume might be evaluated by the following expression:

$$
P=1.65 J * n \mathrm{~W} / \mathrm{cm}^{2},
$$

where $J-$ the flux of thermal neutrons in the units $10^{14} \mathrm{~cm}^{-2} \mathrm{c}^{-1}$, and $n$ - isotope pressure in ${ }^{3} \mathrm{He} \mathrm{atm}$.

To ensure uniform volume ionization it is needed to choose in the reactive zone of reactor some definite place with the homogeneous neutron flux distribution. Taking to the concentration the vertical and horizontal neutron flux distribution within the central diagnostic channel of the WWR-K reactor (Figure 2) the area within diameter $30 \mathrm{~mm}$ and the high $h=300 \mathrm{~mm}$ (from $-150 \mathrm{~mm}$ to $+150 \mathrm{~mm}$ ) was chosen to run the planned experiments and it is corresponding to the most suitable uniform neutron flux distribution. Following to this preferences the ampules size was taking around the $d \leq 30 \mathrm{~mm}, h \leq$ $300 \mathrm{~mm}$. Nuclear reactions in ionising testing gas should be chosen due to the following conditions: sufficiently enough fission cross section and large value of released energy of its products.

Following to the internal WWR-K constructional elements and specific features of the reactor experiments the special construction of diagnostic channel was designed to insure effective radiation of testing gases in the centre of active zone. The probe diagnostic experimental channel to study plasma created by the products of nuclear reactions is schematically presented in the Figure 1. The diagnostic channel is made from the steel tube. At the same picture the diagnostic ampule is presented which has the cylindrical form with the diameter $40 \mathrm{~mm}$, at the bottom of which the special inlet was attached to fill into the cell testing gas mixture of a given composition. At the top side of the ampule the special vacuum sealed entry was installed at the tips of which the cylindrical, spherical and plane probe are attached. The probes are fixed on the ceramic holders. Not operating part of the probe is protected from the plasma contact by isolator made from ceramic tubes. Heating, pumping and gas filling of the testing mixture were conducted on the high vacuum experimental set $\left(10^{-11}\right.$ Torr $)$.
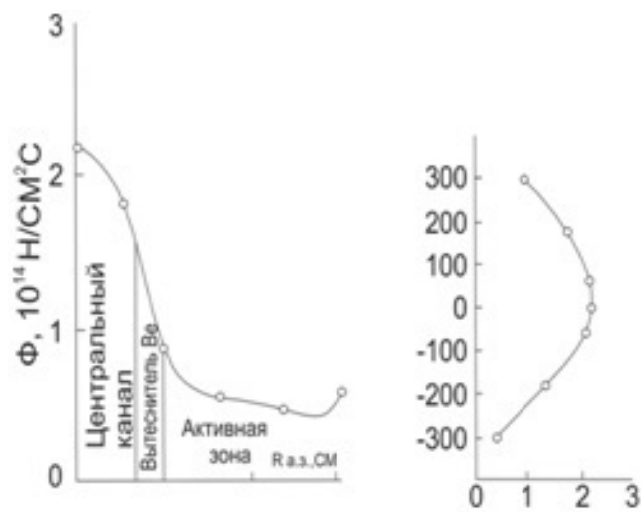

Figure 2 - Space energy distribution of the thermal neutron fluxes along the vertical and horizontal projections within the active zone of WWR-K reactor

The interaction of electrons, ions, neutrals and fission fragments with the probe wall different physical phenomena are taking place that namely the scattering of incident particles, secondary electronic emission, cathode spraying and absorption processes and thin film creation. With the temperature (higher then $800 K$ ) these processes are associated with the evaporation neutral and charged particles from the metal surface and thermo-electronic emission is appearing. The secondary electronic emission initiated by the positive ions, photons and metastable atoms collisions against the metal surface of the probe and has sufficient influence on the formation electric currents on the negative probe. Secondary emission will cause the increase of the ion electric currents on the probe. The positive probe is not so influenced by the secondary electrons due to the slowing electric field.

The number of secondary electrons created by the incident ion striking the surface of the metal is called the secondary emission coefficient $\gamma$ [6]. The value of the $\gamma$ is defined most of all by the kinetic energy of the incident particles the physical and chemical structure of the metal surface.

The value of the $\gamma$ within the region $10^{-1}-10^{-2}$. The emission current density caused by nuclear 
fragments interactions with the wall might be evaluated from the following expression:

$$
J=\frac{e N_{i} \bar{v}_{i}}{4} \gamma_{e i}
$$

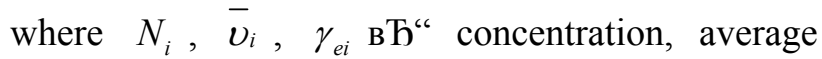
velocity, secondary emission coefficient of the fission fragments, ions or excited atoms.

The secondary electrons emission also might be caused by photons and the density of the electric current emission caused by the electromagnetic radiation is defined by expression:

$$
J=\frac{e N_{i}^{*}}{4 \pi \mu_{\Phi}} \gamma_{\Phi},
$$

where $N^{*}$ - excited atoms concentrations emitting

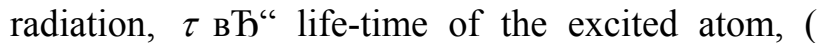
$\tau=10^{-7}-10^{-8} s \quad, \quad \mu_{\Phi} \quad-$ photon absorption coefficient $\left(\mu_{\Phi} \approx 10^{2}\right), \quad \gamma_{\Phi}-$ photoelectrons quantum output $\left(\gamma_{\Phi} \approx 10^{-4}\right)$. Evaluations show that the emission electric current is less than $-<10^{-8}$ $\mathrm{Acm}^{-2}$.

The isolating properties of ceramic tubes in the centre of active zone are not so sufficiently changed and value of electric current leakage might be neglected compared with the electric current on the probe. The evaluation shows that density of emission current $<10^{-8} \mathrm{Acm}^{-2}$ while the density of measured electric current of the probe is not less then $<10^{-6}$ $\mathrm{Acm}^{-2}$. To avoid the cathode spray effect on the CVC the probes are made from refractory metals like wolfram, stainless steel. Not operating part of the probe is protected by isolator which has the following composition: $S i \sim 47 \%, A l 2 O 3 \sim 22 \%$, $\mathrm{Fe} 2 \mathrm{O} 3 \sim 5.7 \%, \quad \mathrm{CaO} \sim 2 \%, \quad \mathrm{MgO} \sim 3 \%$, $K 2 O \sim 3 \% \quad, \quad N 2 O \sim 1.5 \% \quad, \quad S O 3 \sim 0.41 \%$, $H 2 O \sim 0.02 \%$.

3.1Probe diagnostics of the plasma of gaseous mixture ${ }^{3} \mathrm{He}: \mathrm{N}_{2}: \mathrm{O}_{2}$

For different geometry of the probe measurements in variety of density of the testing gas and neutron flux $1-\Phi=1.510^{12} \mathrm{~cm}^{-2} \mathrm{~s}^{-1}, 2-\Phi=3.0$ $\cdot 10^{\Phi} 12 \mathrm{~cm}^{-2} \mathrm{~s}^{-1}$ are presented on the figure $3,4,5$. The results of the experiments in plasma ${ }^{3} \mathrm{He}: \mathrm{N}_{2}: \mathrm{O}_{2}$ are presented in the Table 1, where the formulas (17-18) have been used.

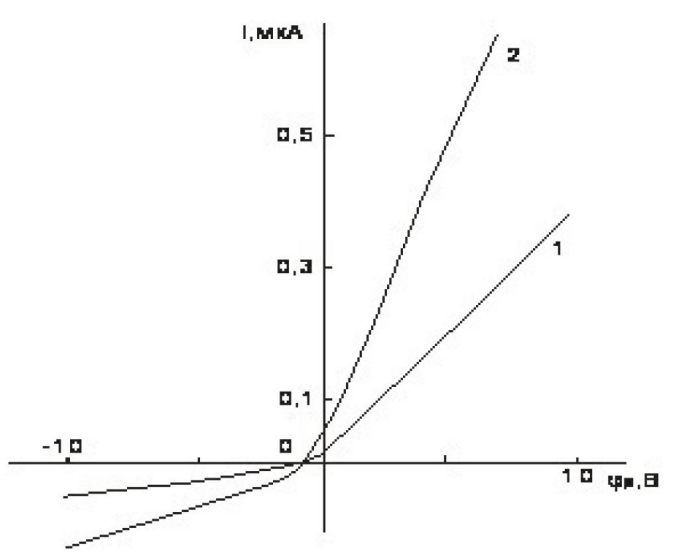

Figure 3 - CVC of cylindrical probe in plasma

$$
\begin{gathered}
{ }^{3} \mathrm{He}: N_{2}: O_{2}(760: 1: 4 \text { Torr }) 1-\Phi= \\
=1.5 \cdot 10^{12} \mathrm{~cm}^{-2} s^{-1}, 2-\Phi=3.0 \cdot 10^{\Phi} 12 \mathrm{~cm}^{-2} s^{-1}
\end{gathered}
$$

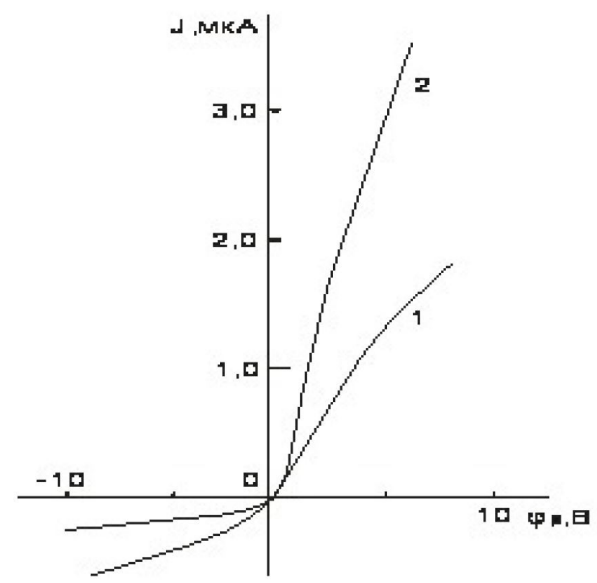

Figure $4-\mathrm{CVC}$ of cylindrical probe in plasma ${ }^{3} \mathrm{He}: \mathrm{N}_{2}: \mathrm{O}_{2}(760: 1: 4$ Torr $) 1-\Phi=$ $=1.5 \cdot 10^{13} \mathrm{~cm}^{-2} s^{-1}, 2-\Phi=3.0 \cdot 10^{\Phi} 13 \mathrm{~cm}^{-2} s^{-1}$

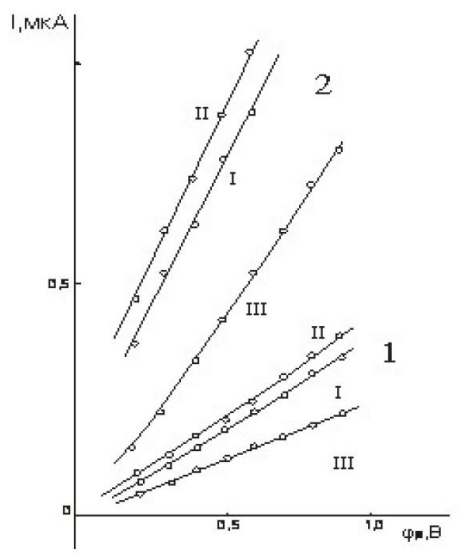

Figure 5 - electronic CVC branches

${ }^{3} \mathrm{He}: \mathrm{N}_{2}: \mathrm{O}_{2}(760: 1: 4$ Torr $) 1-\Phi=$

$$
=1.5 \cdot 10^{12} \mathrm{~cm}^{-2} s^{-1}, 2-\Phi=3.0 \cdot 10^{\Phi} 12 \mathrm{~cm}^{-2} s^{-1} \cdot
$$

1 - cylindrical probe, 2 - spherical probe, 3 - plane probe 
The values of the electron and ion concentrations obtained from the experimental CVC measurements are presented in the Table 1.
The presented results are in satisfactory agreement between each other and compared with the theoretical calculations.

Table 1 - The charged particles concentrations obtained from experimental measurements in plasma of gaseous mixture ${ }^{3} \mathrm{He}-\mathrm{N}_{2}-\mathrm{O}_{2}$

\begin{tabular}{|c|c|c|c|c|c|c|c|}
\hline Reactorpower & $\begin{array}{c}\text { Gas temperature, } \\
K\end{array}$ & Probe & $\begin{array}{c}\text { Probe current } \\
10^{-7} A\end{array}$ & $\begin{array}{c}n^{+} 10^{10} \mathrm{~cm}^{-3} \\
{[11]}\end{array}$ & $\begin{array}{c}n^{+} 10^{10} \mathrm{~cm}^{-3} \\
{[11]}\end{array}$ & $\begin{array}{c}\sigma^{\infty} 10^{-6} \\
\mathrm{Ohm}^{-1} \mathrm{~cm}^{-1}\end{array}$ & $\begin{array}{c}n^{e} 10^{10} \mathrm{~cm}^{-3} \\
{[11]}\end{array}$ \\
\hline & & I & 0.7 & 1.7 & 1.8 & 36.6 & 1.4 \\
\hline 200 & 340 & II & 0.4 & 1.8 & 1.8 & 35.1 & 1.4 \\
\hline & & I & 1.8 & 2.9 & 2.8 & 66.1 & 2.4 \\
\hline 500 & 350 & II & 0.8 & 2.7 & 3.0 & 64.2 & 2.5 \\
\hline & & I & 4.6 & 5.2 & 3.9 & 87.1 & 4.0 \\
\hline 800 & 375 & II & 2.5 & 5.5 & 4.2 & 90.4 & 4.3 \\
\hline & & I & 7.0 & 6.2 & 4.5 & 105.0 & 5.0 \\
\hline
\end{tabular}

CVC of the semispherical $(R=0.1 \mathrm{~cm})$ and plane probe $(R=0.1 \mathrm{~cm})$, were measured in the helium plasma of special purity which was equal to $0.0001 \%$. Pumping, heating and an ampule filling was accomplished on the high level vacuum experimental set. ${ }^{3} \mathrm{He}$ plasma of the pressure 430Torr was studied under the different power levels $200,500,800,100 \mathrm{~kW}$., which corresponded to the neutral flux values $\Phi=(6,15,24,30) * 10^{11} \mathrm{~cm}^{-2} \mathrm{~s}^{-1}$. The temperature was measured by thermocouple device and was equal to $340,350,373,400 \mathrm{~K}$ which corresponded to each previously mentioned power level of reactor.
The typical probe CVC measured in plasma created in the active zone of reactor are presented in the Figure (6-8).

As it might be seen from this pictures in the region not so large potentials of the probe $(0 \div 0.1) B$ it is possible to distinguish linear part of CVC.

The values of conductivity obtained from the linear part of CVC probe of different geometrical configuration are presented in the Table 2. As it follows from the Table 2 the maximum experimental error is about $20 \%$.

Table 2 - The conductivity values obtained from the experiment ${ }^{3} \mathrm{He}-\mathrm{N}_{2}-\mathrm{O}_{2}$

\begin{tabular}{|c|c|c|c|c|c|}
\hline Power mW & Probe & Cell's number [1] & Cell's number [2] & Cell's number [3] & Cell's number [7] \\
\hline & & $\sigma_{\infty} 10^{-6} \mathrm{Ohm}^{-1} \mathrm{~cm}^{-1}$ & $\sigma_{\infty} 10^{-6} \mathrm{Ohm}^{-1} \mathrm{~cm}^{-1}$ & $\sigma_{\infty} 10^{-6} \mathrm{Ohm}^{-1} \mathrm{~cm}^{-1}$ & $\sigma_{\infty} 10^{-6} \mathrm{Ohm}^{-1} \mathrm{~cm}^{-1}$ \\
\hline & 1 & 0.5 & 0.4 & 0.6 & 0.5 \\
\hline 0.5 & 2 & 0.6 & 0.5 & 0.7 & 0.6 \\
\hline & 3 & 0.7 & 0.7 & 0.8 & 0.7 \\
\hline & 1 & 1.4 & 1.2 & 1.5 & 1.4 \\
\hline 1.0 & 2 & 1.4 & 1.2 & 1.9 & 1.4 \\
\hline & 3 & 1.9 & 1.6 & 1.8 & 1.8 \\
\hline
\end{tabular}

\subsection{Uranium hexafluoride plasma probe} diagnostics $\left(U F_{6}\right)$

The experimental study of high pressure $U F_{6}$ plasma created by a volume source of fission fragments is a part of large scientific programs connected with the nuclear energy transformation in other different application forms. The $U F_{6}$ probe diagnostics in the active zone of WWR-K are presented on the Figures (6-9). Uranium hexafluoride $U F_{6}$ also might be used as a fuel in nuclear reactor [7].

In spite of definite number of obstacles which are coming dealing with materials like the fuel of nuclear 
reactors as well as due to it is high chemical aggression the future prospects of $U F_{6}$ use and applications are very attractive.

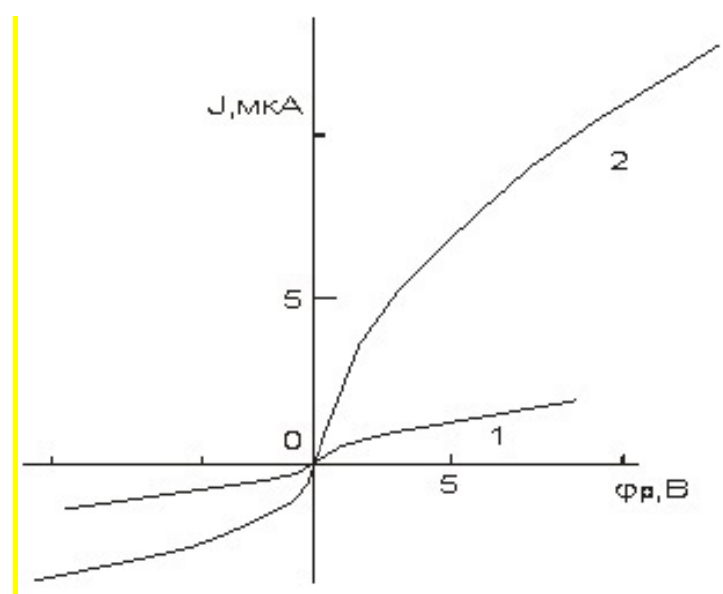

Figure 6-CVC of $U F_{6}$ plasma measured under the different levels of the nuclear WWR-K power $\left(1-1.1012 n / \mathrm{cm}^{2} s-2 s, 2-11013 n / \mathrm{cm}^{2} s-2 s\right)$

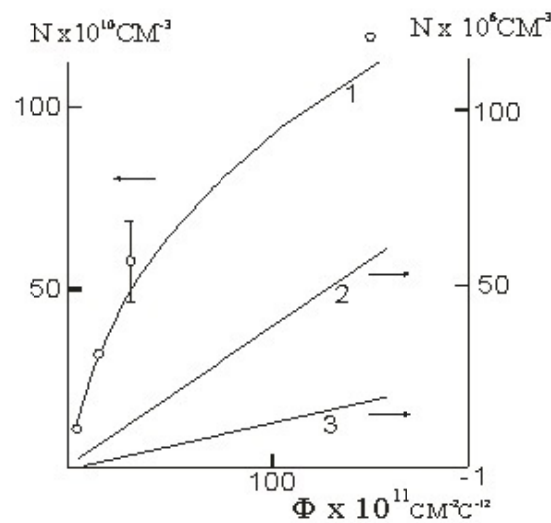

Figure 8 - The charged particle concentrations dependences on the neutron flux level (- calculations; 0 -

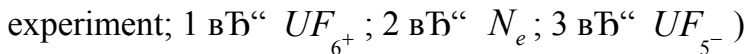

The calculations of the charges particle concentrations in the uranium hexafluoride $U F_{6}$ are presented in the Table 3 [7].

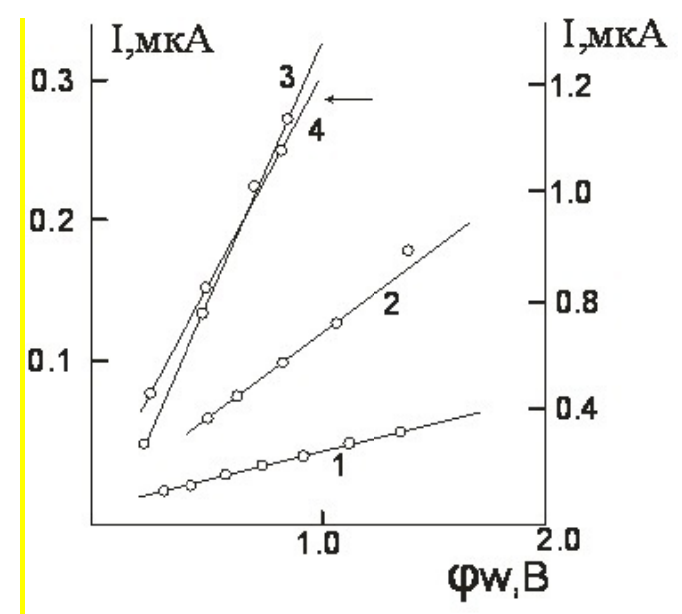

Figure 7-CVC of $U F_{6}$ plasma measured under the different levels of the nuclear WWR-K power (small positive potentials)

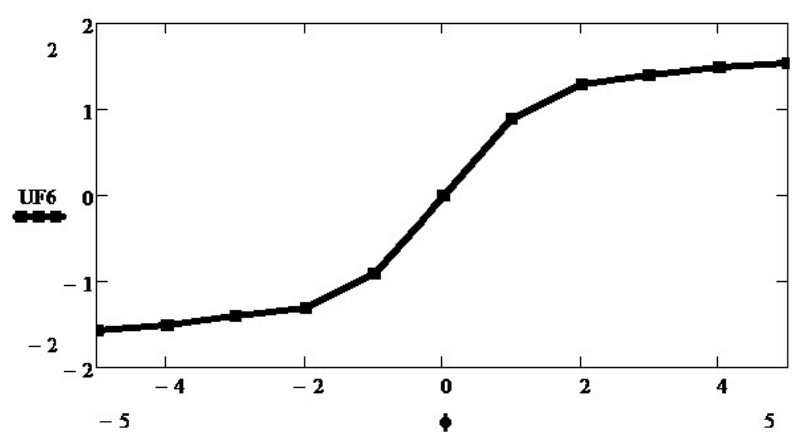

Figure $9-\mathrm{CVC}$ of $U F_{6}$ plasma (typical CVC)

Table 3 - Uranium hexafluoride $U F_{6}$ component concentrations, $\mathrm{cm}^{-3}$

\begin{tabular}{|c|c|c|c|c|}
\hline$N_{e} 5.9 * 10^{6}$ & $U F_{6^{+}} 2.6 * 10^{11}$ & $U F_{6^{-}} 2.6 * 10^{11}$ & $U F_{5^{-}} 2.1 * 10^{6}$ & $F^{-} 4.2 * 10^{4}$ \\
\hline$F 7.7 * 10^{13}$ & $F_{2} 7.7 * 10^{16}$ & $U F_{5} 3.9 * 10^{14}$ & $U F_{4} 4.0 * 10^{4}$ & \\
\hline
\end{tabular}

As it follows from the Table 3, the major part of negative ions in the given mixture is $U F_{6^{-}}$. This concentration is equal to the concentration $U F_{6^{+}}$ with the accuracy of $0.0001 \%$. The concentrations of the ions $U F_{6^{+}}$defined by ionization and recombination rates with $U F_{6^{-}}$. The electron concentration is relatively small. As the theoretical calculations show that the ratio of electrons density 
to the density of negative ions is equal to $10^{-4}$. Also it follows from theoretical calculations that the electron concentration is defined by ionization rate and affinity rate to molecules $U F_{6}$.

\section{Conclusion}

1. The precise experimental CVC measurements of the plane and spherical probe in helium plasma are carried ut within the pressure of the gas 430 Torr and the nuclear reactor power level under 100, 200, 500,
$800,1000 \mathrm{~kW}$. Electron concentrations are defined by used the linear CVC dependence of the positive probe [5].

2. The uranium hexafluoride CVC measurements are also carried out. It was found that at the nuclear power levels 100-200 $\mathrm{kW}$ negative and positive parts of the $U F_{6} \mathrm{CVC}$ are symmetric ones. From the CVC symmetry it might be concluded that the electrons influence on the plasma conductivity is very small $b^{-} n^{-} \gg b_{e} n_{e}$ but $b^{-} \ll b_{e}$, then $n_{-} \gg n_{e}$

\section{References}

[1] M. Benilov. Teoriya electricheskih zondov v potokah slaboionizirovannoi plazmy vysokogo davleniya. Teplofizika vysokih temperatur. -1988. - Vol. 26. - P. 5.

[2] M. Bektursunova. S. Kunakov. Asimptoticheskaya teoriya sfericheskogo electrostaticheskogo zonda $\mathrm{v}$ stolknovitelnoi plazme. obrazovannoi produktami yadernyh reakcyi. Teplofizika vysokih temperatur. - 1992. - Vol.30. - P. 2.

[3] S.K. Kunakov. E.Ye. Son. A. Ye. Shapiyeva.
Probe diagnostics of $3 \mathrm{He}+\mathrm{UF} 6$ plasma. generated in the core of nuclear reactor WWW-K // International Journal of Mathematics and Physics. - 2015. - Vol. 6. - No.1. - P. 69-74.

[4] I. Bronshtein. B.Fraiman. Vtorichnaya electronnaya emissiya. - Moscow, 1969.

[5] V. Dmitrievski. Ye. Voinov. S. Tetelbaum. Primenenie UF6 v yadernyh energeticheskih ustanovkah // Atomnaya energiya. - 1970. - N.4. - Vol. 29. - P. 45-52. 